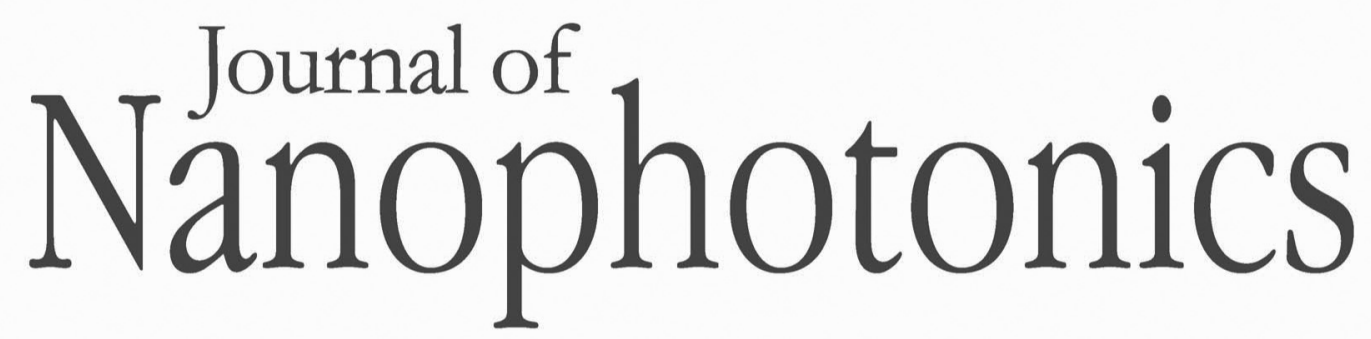

\title{
Special Section Guest Editorial: Metamaterials and Photonic Nanostructures
}

S. Anantha Ramakrishna

Gopinathan Unnikrishnan

Ramarao Vijaya 


\title{
Special Section Guest Editorial: Metamaterials and Photonic Nanostructures
}

\author{
S. Anantha Ramakrishna, ${ }^{\mathrm{a}}$ Gopinathan Unnikrishnan, ${ }^{\mathrm{b}}$ and \\ Ramarao Vijaya ${ }^{\mathrm{a}, \mathrm{c}}$ \\ ${ }^{\mathrm{a}}$ Department of Physics, Indian Institute of Technology Kanpur, Kanpur 208016, India \\ ${ }^{b}$ Photonics Division, Instruments Research and Development Establishment, DRDO, \\ Raipur Road, Dehradun 248008, India \\ ${ }^{\mathrm{c}}$ Center for Lasers and Photonics, Indian Institute of Technology Kanpur, Kanpur 208016, India
}

Over the past decade, scientists have learned how to manipulate the interaction of radiation with structured materials to an unprecedented level. For decades, our world was limited to materials with primarily positive permittivities and permeabilities, with a few exceptions such as plasmas, for example, whose permittivities can be negative. The research in metamaterials coupled with the rapid advancements in micro- and nanofabrication technologies has removed this limitation and has opened the door to almost arbitrary material properties with some extraordinary consequences across the electromagnetic spectrum, ranging from radio frequencies to optical frequencies. These developments have been strongly complemented by the developments in periodic dielectric photonic crystals where band-gap effects lead to mind-blowing dispersion characteristics and consequent effects. Almost simultaneously, the optics of metals has been brought to center-stage where the plasmonic excitations enable the electromagnetic radiation to interact resonantly with the structured material at very small sub-wavelength scales. These interactions prove to be the key to manipulating the optical near-field, and surprising effects such as sub-wavelength scale imaging by so-called super-lenses made of negative refractive index or plasmonic materials have been discovered.

A focused discussion meeting on Metamaterials and Photonic Nanostructures was held at IIT Kanpur on 16-17 August 2013 to bring researchers working on these topics to a common platform and to enable an interactive period for knowledge pooling. One of the intents was to take stock of the work carried out in India in these frontier areas, and most of the active researchers in India contributed to the meeting. This special section consists of selected papers based on work presented at the meeting, as well as papers contributed in response to the call for this special section.

The special section has attracted an interesting collection of works ranging from surface enhanced Raman scattering from nanostructured plasmonic systems, interactions between localized and propagating surface plasmons in subwavelength gratings, extraordinary transmission in structured plasmonic thin films, anisotropic uniaxial metamaterials and epsilon-zero metamaterials, fabrication techniques for embedding nondiffracting defect sites, large area nanophotonic structures and inorganic-organic layered perovskites, to theoretical treatments of third harmonic generation in photonic crystals and super-continuum generation in microstructured optical fibers. The large variety of the topics is reflective of the ever-increasing appeal and applications of metamaterials and nanostructured materials across optics.

\section{Acknowledgments}

The guest editors thank all of the authors for their contributions and the program committee of the meeting for guidance. In particular, Dr. A.K. Gupta from IRDE Dehradun and Prof I. Manna from IIT Kanpur are thanked for their encouragement.

S. Anantha Ramakrishna joined IIT Kanpur in 2003 as an assistant professor and is presently a professor of physics. He obtained his MSc in physics from IIT Kanpur in 1995 and his PhD at the Raman Research Institute, Bangalore. He has coauthored over 62 peer-reviewed papers in journals and a research monograph titled Physics and Applications of Negative Refractive Index Materials. He was awarded the Young Scientist medal of the Indian National Science 
Academy in 2007 and the Swarna Jayanti Fellowship (DST, India) in 2012. His present research interests concern metamaterials and plasmonic nanostuctures.

Gopinathan Unnikrishnan is a scientist with Instrument Research and Development Establishment, Dehradun. He obtained his PhD from the Indian Institute of Technology, Delhi. He has 23 peer-reviewed journal papers. His research interests include the application of computational imaging, compressive sensing and sparse representation techniques to enhance the performance of imaging systems.

Ramarao Vijaya received her master's and doctoral degrees in physics from IIT Madras. She worked at IIT Bombay for more than 13 years before moving to IIT Kanpur, where she is presently a professor. Her research interests are in the areas of nonlinear optics, fiber optics, and photonic crystals. She is a senior member of IEEE and a member of OSA, SPIE and the Indian Laser Association, in all of which she has held executive positions. 